\title{
Influências do trabalho noturno no sono dos trabalhadores de enfermagem: revisão integrativa
}

\author{
Influences of night shifts in sleeping of nursing workers: \\ integrative review
}

\author{
Bruna Karen Cavalcante Fernandes ${ }^{1}$ Adna Cynthya Muniz Ribeiro ${ }^{2}$ Cintia Lira Borges ${ }^{3}$ - Francisca Tereza de Galiza ${ }^{4}$ \\ Emanuella Silva Joventino ${ }^{5}$
}

\begin{abstract}
RESUMO
Objetiva-se identificar as influências do trabalho noturno no sono dos trabalhadores de enfermagem. Trata-se de uma revisão integrativa, realizada em outubro de 2015, nas Bases de dados: Literatura Latino-Americana e do Caribe em Ciências da Saúde (LILACS), Literatura Internacional em Ciências da Saúde (MEDLINE) and Banco de Dados de Enfermagem (BDENF), utilizando os Descritores em Ciências da Saúde: Sono; Enfermagem e Trabalho noturno. Foram incluídos artigos sobre a temática; de livre acesso; nos idiomas: inglês, português e espanhol; publicados na íntegra. Não houve recorte temporal. A amostra foi constituída de 13 artigos. Emergiram duas principais categorias: Qualidade do sono dos trabalhadores de enfermagem do turno noturno; e Repercussões do trabalho noturno na saúde do trabalhador de enfermagem. Concluí-se que o trabalho noturno tem influência direta e indireta sobre a saúde do profissional, pois trabalhar no sentido inverso ao funcionamento fisiológico do organismo pode levar a alterações como distúrbios psíquicos, cardiovasculares e gastrintestinais.
\end{abstract}

Palavras-chave: Enfermagem; Sono; Trabalho Noturno; Saúde do Trabalhador.

\section{ABSTRACT}

The aim is to identify the influences of night work in the sleep of nursing staff. This is an integrative review held in October 2015, in databases: Literatura Latino-Americana e do Caribe em Ciências da Saúde (LILACS), Literatura Internacional em Ciências da Saúde (MEDLINE) and Banco de Dados de Enfermagem (BDENF) using the Descriptors in Health Sciences: Sleep; Nursing and night work. They included articles on the subject; free access; in the languages: English, Portuguese and Spanish; published in full. There was no time frame. The sample consisted of 13 articles. Emerged two main categories: sleep quality of nursing workers in the night shift; and Repercussions of night work on the health of nursing workers. It concludes that night shifts have direct and indirect influence on the health of the professional, since working in the opposite direction to the physiological functioning of the organism can lead to alterations such as psychic, cardiovascular and gastrointestinal disorders.

Keywords: Nursing; Sleep; Night Shift; Worker's health. 


\section{INTRODUÇÃO}

A Enfermagem é uma atividade profissional, desempenhada por enfermeiro, técnico e auxiliar de enfermagem, que destinam sua assistência à pessoa, à família ou à comunidade e que, dependendo do cenário de atuação, realiza um trabalho ininterrupto durante as 24 horas do dia. Ao exercer suas atividades em turnos diurnos e noturnos, o profissional precisa forçar o organismo a adaptar-se a essa situação imposta, em que se inverte o ciclo vigília-sono ${ }^{1}$.

Considerando-se os trabalhadores da equipe de enfermagem que cumprem escalas com plantões noturnos em um período que, de acordo com o ciclo circadiano, a pessoa deveria estar em repouso, sabe-se que essas jornadas de trabalho, sobretudo quando prolongadas, podem contribuir para o aumento das queixas de sono com implicações significativas na vida pessoal e profissional. Estudiosos afirmam que dormir é tão importante para manter a saúde quanto ter uma alimentação saudável².

0 trabalho noturno altera os períodos de sono e vigília, transgredindo as regras do funcionamento fisiológico humano. Além disso, também desencadeia as sensações de mal-estar, fadiga, flutuações no humor, reduções no desempenho devido ao déficit de atenção e concentração e pode, ainda, provocar distúrbios gastrointestinais, cardiovasculares, entre outros ${ }^{3}$.

A legislação brasileira prevê a obrigatoriedade de um intervalo de uma a duas horas para repouso ou alimentação nos casos de trabalho contínuo (ao longo de 24 horas) cuja jornada exceda seis horas ${ }^{4}$. No entanto, a permissão informal para dormir ou repousar por até três horas durante a jornada noturna é comum entre as equipes de enfermagem de hospitais públicos ${ }^{5}$.

Destaca-se, ainda, a política oficial da Saúde do Trabalhador, que tem como objetivo a promoção e a proteção da saúde dos trabalhadores, pelo desenvolvimento de ações de vigilância dos riscos e condições ambientais de trabalho e dos agravos à saúde, promovendo a prestação da assistência, tratamento e reabilitação de forma integrada por meio de uma atuação multiprofissional, interdisciplinar e intersetorial ${ }^{6}$.

Nesse contexto, objetivou-se identificar as influências do trabalho noturno no sono dos trabalhadores de enfermagem.

\section{MÉTODO}

Estudo bibliográfico de caráter descritivo utilizando o método da revisão integrativa da literatura para coleta e análise dos dados. A revisão integrativa da literatura é um método de pesquisa que possibilita a síntese do estado do conhecimento de uma determinada temática, identificando lacunas existentes para sugestões de novos estudos e perspectivas da temática estudada ${ }^{7}$. Para tanto, foram observadas as seguintes etapas: elaboração da pergunta norteadora; busca ou amostragem na literatura; coleta de dados; análise crítica dos estudos incluídos; discussão dos resultados e apresentação da revisão/síntese do conhecimento ${ }^{8}$.

Os dados foram coletados em outubro de 2015 e para o levantamento dos mesmos foi utilizada como bases de dados: Literatura Latino-Americana e do Caribe em Ciências da Saúde (LILACS), Literatura Internacional em Ciências da Saúde (MEDLINE) e Base de Dados de Enfermagem (BDENF). Como estratégias de busca, foram utilizados descritores de assunto contidos nos Descritores em Ciências da Saúde - DeCS, a saber: Sono, Enfermagem, Trabalho noturno. Os descritores foram combinados nas seguintes formas: Sono AND Enfermagem; Sono AND Trabalho Noturno; Enfermagem AND Trabalho Noturno.

Delineou-se como critérios de elegibilidade: estudos completos sobre o sono e o trabalho noturno dos trabalhadores de enfermagem; de livre acesso; em idioma inglês, português e espanhol; publicados na íntegra. Foram excluídos os estudos em duplicidade nos bancos de dados, de revisão, teses e dissertações e os artigos que, de alguma forma, não respondiam à questão norteadora da pesquisa. Não houve recorte temporal nas publicações dos artigos.

Com o intuito de se esgotar todas as possibilidades de busca, os descritores foram combinados entre si, resultando no total de 2373 publicações encontradas e destas apenas 28 contemplavam a questão norteadora. Salienta-se que 12 estudos apresentavam duplicidade e três não estavam disponíveis na íntegra. Portanto, apenas 13 artigos atendiam aos critérios de inclusão, os quais constituíram a amostra final da pesquisa, sendo: nove na LILACS, três na MEDLINE e um na BDENF.

Após a seleção dos artigos, foi realizada uma primeira leitura das publicações completas para verificar sua adequação aos objetivos propostos neste estudo. Os mesmos foram fichados, buscando-se identificar suas principais abordagens em relação ao trabalho noturno e sua relação com o sono dos trabalhadores de enfermagem e as possíveis lacunas existentes na literatura.

Realizou-se uma análise descritiva dos artigos selecionados, através de um instrumento previamente elaborado que contemplava os itens: identificação do artigo, tipo de publicação, características metodológicas do estudo, resultados encontrados e conclusões.

Os estudos foram analisados criticamente por meio de leitura na íntegra. Após análise, foi realizada uma síntese dos estudos selecionados sendo organizados em temas, observando as suas confluências e divergências gerando duas categorias: qualidade do sono dos trabalhadores de enfermagem do turno noturno; repercussões do trabalho noturno na saúde do trabalhador de enfermagem.

\section{RESULTADOS}

A amostra do estudo foi composta por 13 artigos, cujos principais dados de caracterização tais como autoria, título e objetivo da publicação, periódico e ano, estão apresentados no quadro 1. 
Quadro 1. Distribuição dos estudos sobre a influência do trabalho noturno no sono dos trabalhadores de enfermagem segundo autores, título, objetivo, periódico e ano de publicação

\begin{tabular}{|c|c|c|c|}
\hline Autores & Título & Objetivo & Periódico/Ano \\
\hline $\begin{array}{l}\text { Palermo TAC, Rotenberg L, } \\
\text { Zeitoune RCG, Silva-Costa } \\
\text { A, Souto EP, Griep RH }\end{array}$ & $\begin{array}{l}\text { Cochilo durante o plantão noturno } \\
\text { e a recuperação após o trabalho } \\
\text { entre enfermeiros de hospitais }\end{array}$ & $\begin{array}{l}\text { Analisar a associação entre duração } \\
\text { do cochilo durante o plantão noturno } \\
\text { e recuperação após o trabalho, entre } \\
\text { enfermeiros. }\end{array}$ & $\begin{array}{l}\text { Revista Latino } \\
\text { Americana de } \\
\text { Enfermagem } \\
\quad 2015\end{array}$ \\
\hline $\begin{array}{l}\text { Palhares VC, Corrente JE, } \\
\text { Matsubara BB }\end{array}$ & $\begin{array}{l}\text { Association between sleep quality } \\
\text { and quality of life in nursing } \\
\text { professionals working rotating } \\
\text { shifts. }\end{array}$ & $\begin{array}{l}\text { To analyze the association between sleep } \\
\text { quality and quality of life of nursing } \\
\text { professionals according to their work } \\
\text { schedules }\end{array}$ & $\begin{array}{l}\text { Revista de Saúde } \\
\text { Pública } \\
2014\end{array}$ \\
\hline Oliveira B, Martino MMF & $\begin{array}{l}\text { Análise das funções cognitivas e } \\
\text { sono na equipe de enfermagem } \\
\text { nos turnos diurno e noturno. }\end{array}$ & $\begin{array}{l}\text { Analisar a memória de curto prazo, a } \\
\text { atenção e o padrão do sono na equipe de } \\
\text { enfermagem nos turnos diurno e noturno. }\end{array}$ & $\begin{array}{l}\text { Revista Gaúcha } \\
\text { de Enfermagem } \\
2013\end{array}$ \\
\hline $\begin{array}{l}\text { Silva-Costa A, Rotenberg L, } \\
\text { Griep RH, Fischer FM }\end{array}$ & $\begin{array}{l}\text { Cochilos durante o trabalho } \\
\text { noturno em equipes de } \\
\text { enfermagem: possíveis benefícios à } \\
\text { saúde dos trabalhadores. }\end{array}$ & $\begin{array}{l}\text { Caracterizar o cochilo durante plantões } \\
\text { noturnos em termos da duração, eficiência, } \\
\text { alocação e qualidade entre trabalhadores de } \\
\text { enfermagem. }\end{array}$ & $\begin{array}{c}\text { Revista da Escola } \\
\text { Anna Nery } \\
2015\end{array}$ \\
\hline $\begin{array}{l}\text { Fischer FM, Teixeira LP, } \\
\text { Borges FNS, Gonçalves } \\
\text { MBL, Ferreira RM }\end{array}$ & $\begin{array}{l}\text { Percepção de sono: duração, } \\
\text { qualidade e alerta em profissionais } \\
\text { da área de enfermagem }\end{array}$ & $\begin{array}{l}\text { Avaliar a percepção da duração e qualidade } \\
\text { dos episódios de sono nos dias de trabalho e } \\
\text { de descanso, bem com o dos níveis de alerta }\end{array}$ & $\begin{array}{l}\text { Caderno de } \\
\text { Saúde Pública } \\
2002\end{array}$ \\
\hline Silva CAR, Martino MMF & $\begin{array}{l}\text { Aspectos do ciclo vigília-sono } \\
\text { e estados emocionais em } \\
\text { enfermeiros dos diferentes turnos } \\
\text { de trabalho. }\end{array}$ & $\begin{array}{l}\text { Descrever o ciclo vigília-sono de enfermeiro } \\
\text { sem função de seus turnos de trabalho, } \\
\text { identificar alterações no padrão de sono e } \\
\text { seus estados emocionais durante o trabalho. }\end{array}$ & $\begin{array}{c}\text { Revista de } \\
\text { Ciências Médicas } \\
2009\end{array}$ \\
\hline $\begin{array}{l}\text { Maynardes DCD, Sarquis } \\
\text { LMM, Kirchhof ALC }\end{array}$ & $\begin{array}{l}\text { Trabalho noturno e morbidades de } \\
\text { trabalhadores de enfermagem. }\end{array}$ & $\begin{array}{l}\text { Identificar os principais agravos à saúde } \\
\text { desses trabalhadores. }\end{array}$ & $\begin{array}{l}\text { Cogitare } \\
\text { Enfermagem } \\
2009\end{array}$ \\
\hline $\begin{array}{l}\text { Medeiros SM, Macêdo } \\
\text { MLAF, Oliveira JSA, Ribeiro } \\
\text { LM }\end{array}$ & $\begin{array}{l}\text { Possibilidades e limites da } \\
\text { recuperação do sono de } \\
\text { trabalhadores noturnos de } \\
\text { enfermagem. }\end{array}$ & $\begin{array}{l}\text { Investigar as possibilidades/limites da } \\
\text { recuperação do sono das trabalhadoras } \\
\text { de nível médio de enfermagem que } \\
\text { desenvolvem suas atividades laborais no } \\
\text { turno noturno no setor de pronto-socorro em } \\
\text { um hospital público, em Natal, RN. }\end{array}$ & $\begin{array}{l}\text { Revista Gaúcha } \\
\text { de Enfermagem } \\
2009\end{array}$ \\
\hline $\begin{array}{l}\text { Barboza JIRA, Moraes EL, } \\
\text { Pereira EA, Reimão RNAA }\end{array}$ & $\begin{array}{l}\text { Avaliação do padrão de sono dos } \\
\text { profissionais de Enfermagem dos } \\
\text { plantões noturnos em Unidades de } \\
\text { Terapia Intensiva. }\end{array}$ & $\begin{array}{l}\text { Avaliar a qualidade do sono e verificar a } \\
\text { presença de sonolência diurna excessiva dos } \\
\text { profissionais de Enfermagem dos plantões } \\
\text { noturnos das Unidades de Terapia Intensiva } \\
\text { do Instituto Central do Hospital das Clínicas } \\
\text { da Faculdade de Medicina da Universidade } \\
\text { de São Paulo. }\end{array}$ & $\begin{array}{l}\text { Revista Einstein } \\
2008\end{array}$ \\
\hline Martino MMF & $\begin{array}{l}\text { Estudo comparativo de padrões } \\
\text { de sono em trabalhadores de } \\
\text { enfermagem dos turnos diurno e } \\
\text { noturno. }\end{array}$ & $\begin{array}{l}\text { Comparar os padrões de sono de enfermeiros } \\
\text { dos turnos diurno e noturno em um hospital } \\
\text { de Campinas (SP), Brasil. }\end{array}$ & $\begin{array}{c}\text { Revista } \\
\text { Panamericana } \\
\text { de Salud Publica } \\
2002\end{array}$ \\
\hline Mendes SS, Martino MMF & $\begin{array}{l}\text { Trabalho em turnos: estado geral } \\
\text { de saúde relacionado ao sono em } \\
\text { trabalhadores de enfermagem. }\end{array}$ & $\begin{array}{l}\text { Identificar os sintomas referentes ao estado } \\
\text { geral de saúde associado ao trabalho em } \\
\text { turnos de enfermagem e relacioná-los com a } \\
\text { qualidade do sono. }\end{array}$ & $\begin{array}{c}\text { Revista da Escola } \\
\text { de Enfermagem } \\
\text { USP } \\
2012\end{array}$ \\
\hline Rocha MCP, Martino MMF & $\begin{array}{l}\text { O estresse e qualidade de sono do } \\
\text { enfermeiro nos diferentes turnos } \\
\text { hospitalares. }\end{array}$ & $\begin{array}{l}\text { Analisar a relação entre estresse e qualidade } \\
\text { do sono de enfermeiros que atuam em } \\
\text { diferentes setores hospitalares, dos turnos } \\
\text { diurnos e noturnos. }\end{array}$ & $\begin{array}{c}\text { Revista da Escola } \\
\text { de Enfermagem } \\
\text { USP } \\
2010\end{array}$ \\
\hline $\begin{array}{l}\text { Silva RM, Beck CLC, } \\
\text { Magnago TSBSM, } \\
\text { Carmagnani MIS, Tavares } \\
\text { JP, Prestes FC }\end{array}$ & $\begin{array}{l}\text { Trabalho noturno e a repercussão } \\
\text { na saúde dos Enfermeiros. }\end{array}$ & $\begin{array}{l}\text { Apresentar e discutir as alterações na saúde } \\
\text { percebidas por enfermeiros do período } \\
\text { noturno. }\end{array}$ & $\begin{array}{c}\text { Revista da Escola } \\
\text { Anna Nery } \\
2011\end{array}$ \\
\hline
\end{tabular}

Fonte: elaboração feita pelos autores. 
Os artigos apresentaram objetivos essencialmente voltados à caracterização do sono, bem como a qualidade deste referente ao trabalhador de enfermagem do período noturno, em uma esfera tanto de mensuração direta (objetiva/quantificadora) quanto de apresentação de dados subjetivos relacionados aos trabalhadores, por meio do registro de suas percepções referentes às repercussões da má qualidade do sono em sua saúde.

Aidentificação dos principais agravos à saúde causados por essa inversão do ciclo-vigília desses trabalhadores, também foi elencada como objetivo de muitos estudos, assim como a comparação dos padrões de sono entre os trabalhadores do turno noturno e dos demais turnos de trabalho.

Observou-se que oito artigos foram publicados em periódicos de enfermagem, sendo os demais publicados em periódicos médicos ou com enfoque multidisciplinar na área da saúde. Em relação ao ano de publicação, observouse uma maior concentração de artigos publicados no ano de $2009(n=3)$, sugerindo que a relevância do tema está se fazendo perceber pelos pesquisadores, principalmente da área de enfermagem.

Quanto à abordagem, dez estudos eram quantitativos e apenas três possuíam uma abordagem qualitativa. Observa-se, portanto, que houve uma queda nas produções sobre essa temática utilizando a abordagem qualitativa. Isso implica em uma lacuna na compreensão acerca de como o trabalhador enfrenta essa questão do trabalho noturno e suas consequências em sua saúde.

Emergiram-se duas principais categorias a partir da análise dos artigos: qualidade do sono dos trabalhadores de enfermagem do turno noturno; e repercussões do trabalho noturno na saúde do trabalhador de enfermagem.

\section{DISCUSSÃO}

\section{Categoria 1 - Qualidade do sono dos trabalhadores de enfermagem do turno noturno}

Os artigos desta categoria somaram seis e versavam sobre a influência do trabalho noturno na qualidade do sono dos trabalhadores de enfermagem, estando aquele associado com prejuízo importante de componentes da qualidade do sono.

Os estudos trouxeram dados referentes aos seguintes quesitos: a importância dos cochilos no trabalho, a fragmentação do sono e a relação do sono com a qualidade de vida desses trabalhadores.

Sabe-se que os cochilos durante o trabalho noturno são uma forma de lidar com a privação parcial do sono. Definido como qualquer período de sono, inferior a $50 \%$ da duração média do sono noturno, o cochilo durante o plantão noturno está associado à menor sonolência durante o trabalho ${ }^{9}$. À medida que ocorre um débito significativo de horas de sono, com aumento da sonolência, isso pode contribuir em longo prazo para a fadiga ${ }^{10}$, com possíveis repercussões à qualidade da assistência.
Em um contexto de trabalhadores de enfermagem com predominância do gênero feminino, o cochilo noturno pode ter maior relevância para trabalhadoras com sobrecarga de trabalho doméstico, pois estas possuem menos tempo para o sono diurno durante os dias de folga ${ }^{11}$.

Outro ponto importante diz respeito às escalas de plantões de 12 horas, seguidos por 36 ou 60 horas de descanso, possibilitando que os trabalhadores dediquemse a mais de uma atividade produtiva trabalhando em mais de uma instituição, e submetendo-se a longas jornadas de trabalho que podem interferir na disponibilidade de tempo para o sono ${ }^{11}$.

A importância do cochilo durante o plantão noturno na recuperação, após o trabalho, foi demonstrada em estudo no contexto da enfermagem brasileira, que identificou a associação do cochilo com melhor recuperação, desde que a jornada de trabalho doméstico não ultrapassasse 10 horas semanais ${ }^{12}$. Os mesmos autores afirmam que não só a ocorrência do cochilo influencia a recuperação após o trabalho, mas também a sua duração.

Estudos sugerem um efeito benéfico do cochilo noturno, de 2 a 3 horas, em termos de recuperação após o trabalho ${ }^{13}$. De fato, este aspecto merece aprofundamento, pois pode constituir um fator limitante da implementação desta prática no contexto dos cuidados em saúde. Ressaltase, ainda, a importância de oferecer condições adequadas para que o cochilo durante o trabalho noturno aconteça $a^{14}$.

Uma das pesquisas constatou que entre os trabalhadores que referiram dormir mais de 3 horas não se observou chances mais elevadas de recuperação, quando comparados àqueles que não dormiam. A grande variabilidade na duração dos cochilos, observada no grupo que dormia por mais de 3 horas, pode explicar, em parte, estes resultados ${ }^{13}$.

Como proposto nos estudos, não há dúvidas de que a possibilidade de tirar um cochilo é um procedimento organizacional essencial para melhorar as condições de trabalho durante o turno noturno. Tal benefício foi explicitado no estudo sobre as condições ambientais e organizacionais dos cochilos nas equipes de enfermagem ${ }^{15}$. Aspectos como quartos para o descanso noturno, tempo regulamentado, luz, barulho e ventilação foram referidos pelas trabalhadoras de enfermagem como fatores importantes no planejamento e organização dos cochilos no trabalho ${ }^{15}$.

Por desenvolverem suas atividades no momento em que o organismo encontra-se preparado para o descanso, os trabalhadores de enfermagem que trabalham neste turno, apresentam conflitos internos e externos relativos à vida social e ao isolamento ${ }^{16}$. Além disso, as condições de trabalho oferecidas são determinantes no processo de saúde-doença dos enfermeiros ${ }^{17}$.

Neste cenário, ressalta-se que o trabalho noturno encontra-se ligado a diversas necessidades, dentre elas, as de cunho financeiro, com a adequação aos demais vínculos 
empregatícios, interferindo assim na estrutura familiar, como no convívio com os filhos e demais familiares. A literatura apoia esta relação, mostrando que alterações do sono tem significativa correlação com a qualidade de vida ${ }^{18}$.

Portanto, observou-se nas produções captadas o interesse na influência da qualidade de vida do trabalhador noturno, no processo saúde-doença e nas repercussões do trabalho noturno na saúde mental e emocional.

\section{Categoria 2 - Repercussões do trabalho noturno na saúde do trabalhador de enfermagem}

Fizeram parte desta categoria sete dos artigos analisados, os quais versavam sobre as consequências da má qualidade do sono na saúde dos trabalhadores. Os artigos relataram alterações de cunho biológico e psíquico oriundas da má qualidade do sono. Foram referidas alterações de memória, dos níveis de atenção, problemas emocionais e aparecimento de sintomas nos diversos sistemas do corpo.

As consequências da realização do trabalho no período noturno na saúde do trabalhador manifestam-se com alterações do equilíbrio biológico, dos hábitos alimentares, do sono, da perda de atenção, da acumulação de erros, do estado de ânimo e da vida familiar e social ${ }^{16}$.

Com relação aos principais problemas apresentados pelos trabalhadores de enfermagem nos estudos analisados, destacam-se a fadiga, alteração no ciclo sonovigília, alterações gastrintestinais (distúrbio de apetite, sensação de má digestão, azia ou queimação), ganho de peso, irritabilidade, insônia, dores de cabeça, dificuldade de concentração, depressão ou infelicidade, diminuição da autoestima e labilidade de humor 16,18-22.

A sonolência é causada pela liberação, ao anoitecer, do hormônio melatonina pela glândula pineal. Essa glândula tem sua função regulada pela luminosidade do dia. Dessa forma, ao ser liberado na corrente sanguínea, esse hormônio, invariavelmente, induz o sono o qual é essencial e exerce importante papel na consolidação da memória ${ }^{16}$.

Os maus hábitos alimentares também foram referidos nos artigos como algo que interfere no sono, salientandose que, comumente, nos plantões noturnos, costumase ingerir alimentos não saudáveis e, apesar de não ser exclusividade dos trabalhadores do turno noturno, fazem parte dos recursos utilizados pelo trabalhador para se manter acordado ${ }^{16}$.

O descuido com a qualidade da alimentação pode estar relacionado à preferência pela ingestão de alimentos de fácil acesso, como os industrializados, que geralmente apresentam baixa densidade nutricional e substâncias que possibilitam o aparecimento de transtornos digestivos. Esses hábitos podem ocasionar um desequilíbrio na secreção e motilidade gastrointestinal, provocando pirose, transtornos de apetite, dificuldades de digestão, constipação e flatulência nos trabalhadores noturnos quando comparados ao do diurno ${ }^{16}$. No entanto, ao analisar esses fatores, deve-se considerar outros fatores como idade, tabagismo, entre outras características individuais.

Sabe-se que a diminuição do tempo de sono modula o ciclo sono-vigília e pode alterar o comportamento alimentar de diferentes maneiras ${ }^{23}$. Uma hipótese sugere que um maior tempo acordado implica em mais tempo disponível para comer, teoricamente facilitando as oportunidades para o consumo de lanches ou de refeições adicionais ao longo do dia ${ }^{24-25}$. Nesta mesma linha, estudos indicam que a restrição do sono é muito associada a irregularidades da ingestão alimentar, com grande frequência, em indivíduos privados de sono, de comportamentos como "beliscar" em excesso ao longo do dia e maior consumo de alimentos de alta densidade calórica ${ }^{26-27-28}$.

O ganho ponderal também se destaca quando associado à impossibilidade da prática de atividade física, especialmente, no dia posterior ao plantão noturno devido ao cansaço físico e mental. Estudos apontam que o estado nutricional dos profissionais da Enfermagem pode sofrer alterações negativas, o que é demonstrado pela elevada prevalência de sobrepeso e obesidade nessa população ${ }^{29-30-31}$. Portanto, sugere-se que os trabalhadores consumam alimentos mais leves nos plantões noturnos, evitem o açúcar em excesso e frituras, restrinjam o excesso de café e realizem atividades físicas sistemáticas, no intuito de minimizar as repercussões do trabalho em sua saúde.

Diante desse cenário, as consequências do trabalho noturno na saúde devem ser fatores de alerta para gestores, instituições empregadoras, academias, órgãos de pesquisa e para os próprios profissionais de enfermagem, a fim de criar condições e estratégias para promover o conforto dos trabalhadores durante o plantão e possibilitar a segurança do paciente, uma vez que os trabalhadores da enfermagem representam um quantitativo expressivo no cenário de cuidado.

\section{CONCLUSÃO}

O trabalho noturno tem influência direta e indireta sobre a saúde do profissional, pois trabalhar no sentido inverso ao funcionamento fisiológico do organismo pode levar a alterações como distúrbios psíquicos, cardiovasculares e gastrintestinais. Além disso, atinge a vida social e familiar do profissional, dificultando sua participação em atividades sociais e no planejamento de vida.

Apesar da limitação do estudo de apenas apontar as falhas do trabalho noturno, é necessário para o trabalhador compreender sobre os limites do seu corpo e sugerir o desenvolvimento de estratégias pelos gestores, envolvendo não só a organização do trabalho, mas aspectos da fisiologia humana para oferecer condições adequadas no serviço e minimizar os efeitos deste turno árduo de trabalho na saúde dos trabalhadores. 


\section{REFERÊNCIAS}

1. Silva RM, Zeitoune RCG, Beck CLC, Loro MM. Matutino, vespertino ou indiferente? produção do conhecimento sobre o cronotipo na enfermagem. Rev Enferm UFSM [Internet]. 2014 [acesso em 30 jul 2016]; 4(4):835-43. Disponível em: http://dx.doi.org/10.5902/2179769212888

2. Portela LF, Rotenberg L, Waissmann W. Self-reported health and sleep complaints among nursing personnel working under $12 \mathrm{~h}$ night and day shifts. Chronobiol Int. [Internet]. 2004 [acesso em 30 jul 2016]; 21:859-70. Disponível em: http://www.ncbi.nlm.nih.gov/pubmed/15646233

3. Barboza, JIRA, Moraes EL, Pereira EA, Reimão RNAA. Avaliação do padrão de sono dos profissionais de enfermagem dos plantões noturnos em unidades de terapia intensiva. Einstein. [Internet] 2008 [acesso em 30 jul 2016];6(3):296301. Disponível em: http://apps.einstein.br/revista/arquivos/ PDF/927-v6n3aA0927portp 296-301.pdf

4. Brasil. Consolidação das Leis do Trabalho - Decreto-lei 5452/43 | Decreto-lei n. ${ }^{\circ}$ 5.452, de $1^{\circ}$ de maio de 1943.

5. Rotenberg L. Aspectos sociais da tolerância ao trabalho em turnos noturno, com ênfase nas questões relacionadas ao gênero. In: Fischer FM, Moreno CRC, Rotenberg L. Trabalho em turnos e noturno na sociedade 24 horas. São Paulo: Atheneu; 2004.

6. Brasil. Política Nacional de Segurança e Saúde do Trabalhador. Portaria Interministerial $n^{\circ} 800$, de 03 de maio de 2005.

7. Polit DF, Beck CT. Using research in evidence-based nursing practice. In: Polit DF, Beck CT, editors. Essentials of nursing research.Methods, appraisal and utilization. Philadelphia (USA): Lippincott Williams \& Wilkins; 2006. 12:457-94.

8. Souza MT, Silva MD, Carvalho R. Revisão integrativa: o que é e como fazer. Einstein [Internet]. 2010 [acesso em 30 jul 2016]; 8:102-6. Disponível em: http://www.scielo.br/pdf/eins/v8n1/ pt_1679-4508-eins-8-1-0102.pdf

9. Fallis WM, Mcmillan DE, Edwards MP. Napping during night shift: practices, preferences and perceptions of critical care and emergency department nurses. CritCare Nurse [Internet]. 2011 [acesso em 30 jul 2016]; 31(2):1-11. Disponível em: http://dx.doi.org/10.4037/ccn2011710

10. Fischer FM, Teixeira LP, Borges FNS, Gonçalves MBL, Ferreira RM. Percepção do sono: duração, qualidade e alerta em profissionais da área de enfermagem. Cad Saúde Publica [Internet]. 2002 [acesso em 30 jul 2016];18:12611269. Disponível em: http://dx.doi.org/10.1590/S0102$311 \times 2002000500018$

11. Silva-Costa A, Fischer FM, Griep RH, Rotenberg L. Relationship between napping during night shift and household obligations of female nursing personnel. Int J of Gen Med [Internet]. 2013 [acesso em 30 jul 2016]; 6:227-31. Disponível em: http:// dx.doi.org/10.2147/IJGM.S41200

12. Silva-Costa A, Rotenberg L, Griep RH, Fisher FM. Relationship between sleeping on the night shift and recovery from work among nursing workers - the influence of domestic work. J Adv Nurs [Internet]. 2011 [acesso em 30 jul 2016]; 67(5):365-8. Disponível em: http://dx.doi.org/10.1111/j.13652648.2010.05552.x

13. Palermo TAC, Rotenberg L, Zeitoune RCG, Silva-Costa A, Souto EP, Griep RH. Cochilo durante o plantão noturno e a recuperação após o trabalho entre enfermeiros de hospitais. Rev Latino-Am Enferm [Internet]. 2015 [acesso em 30 jul 2016]; 23(1):114-21. Disponível em: http://dx.doi. org/10.1590/0104-1169.0147.2532

14. Silva-Costa A, Rotenberg L, Griep RH, Fisher FM. Cochilos durante o trabalho noturno em equipes de enfermagem: possíveis benefícios à saúde dos trabalhadores. Esc Anna Nery Rev Enferm [Internet]. 2015 [acesso em 30 jul 2016]; 19(1):33-39. Disponível em: http://dx.doi.org/10.5935/14148145.20150005

15. Silva-Costa A, Araújo MM, Nagai R, Fisher FM. Environmental and organizational conditions for napping during night work: a qualitative study among nursing professionals. Sleep Sci [Internet]. 2010 [acesso em 30 jul 2016]; 3(1):11-5. Disponível em:http://www.sleepscience.com.br/pdf/articles/vol3/ SleepScience_vol3_issue01_art03.pdf

16. Silva RM, Carmagnani MIS, Beck CLC, Tavares JP, Magnago TSBS, Prestes FC. Trabalho noturno e a repercussão na saúde dos enfermeiros. Esc Anna Nery [Internet]. 2011 Apr/June [acesso em 25 jul 2016];15(2);270-6. Disponível em: http:// www.scielo.br/pdf/ean/v15n2/v15n2a08

17. Santos AA, Costa ORS. Qualidade de vida no trabalho dos profissionais de enfermagem que atuam no período noturno em um hospital escola do sul de minas gerais. Rev Cien em Saúde [Internet]. 2016 [acesso em 30 jul 2016]; 6(1):43-51. Disponível em: http://186.225.220.234:8484/rcsfmit/ojs2.3.3-3/index.php/rcsfmit_zero/article/view/453

18. Palhares VC, Corrente JE, Matsubara BB. Association between sleep quality and quality of life in nursing professionals working rotating shifts. Rev Saúde Pública [Internet]. 2014 [acesso em 30 jul 2016]; 48(4): 594-601. Disponível em: http://dx.doi.org/10.1590/S0034-8910.2014048004939

19. Maynardes DCD, Sarquis LMM, Kirchhof ALC. Trabalho noturno e morbidades de trabalhadores de enfermagem. Cogitare Enferm [Internet]. 2009 Oct/Dec [acesso em 30 jul 2016]; 14(4):703-8. Disponível em: http://www. revenf.bvs.br/scielo.php?script=sci_arttext $\&$ pid=S141485362009000400014\&lng=es.

20. Mendes SS, Martino MMF. Trabalho em turnos: estado geral de saúde relacionado ao sono em trabalhadores de enfermagem. Rev. esc. enferm. USP [Internet]. 2012 Dec [acesso em 10 ago 2016]; 46(6):1471-1476. Disponível em: http://dx.doi. org/10.1590/S0080-62342012000600026.

21. Oliveira B, Martino MMF. Análise das funções cognitivas e sono na equipe de enfermagem nos turnos diurno e noturno. Rev. Gaúcha Enferm. [Internet]. 2013 Mar [acesso em 10 ago 2016]; 34(1):30-36. Disponível em: http://dx.doi.org/10.1590/ S1983-14472013000100004.

22. Medeiros SM, Macêdo MLAF, Oliveira JSA, Ribeiro LM. Possibilidades e limites da recuperação do sono de trabalhadores noturnos de enfermagem. Rev Gaúcha Enferm. [Internet]. 2009 Mar [acesso em 10 ago 2016]; 30(1):92-8. Disponível em: http://seer.ufrgs.br/index.php/ RevistaGauchadeEnfermagem/article/view/5111/6568

23. Crispim CA, Zalcman I, Dáttilo M, Padilha HG, Tufik S, Mello MT. Relação entre sono e obesidade: uma revisão da Literatura. Arq Bras Endocrinol Metab [Internet]. 2007 [acesso em 08 mar 2017];51(7):1041-9. Disponível em: http://dx.doi. org/10.1590/S0004-27302007000700004 
24. Knutson KL, Spiegel K, Penev P, Van Cauter E. The metabolic consequences of sleep deprivation. Sleep Med Rev [Internet]. 2007 [acesso em 08 mar 2017];11(3):163-78. Disponível em: http://www.ncbi.nlm.nih.gov/pubmed/17442599

25. Hursel R, Rutters F, Gonnissen HK, Martens EA, WesterterpPlantenga MS. Effects of sleep fragmentation in healthy men on energy expenditure, substrate oxidation, physical activity, and exhaustion measured over $48 \mathrm{~h}$ in a respiratory chamber. Am J Clin Nutr. [Internet]. 2011 [acesso em 08 mar 2017];94(3):804-8. Disponível em: http://dx.doi.org/10.3945/ ajcn.111.017632

26. Spiegel K, Leproult R, L'hermite-Baleriaux M, Copinschi G, Penev PD, Van Cauter E. Leptin levels are dependent on sleep duration: relationships with sympathovagal balance, carbohydrate regulation, cortisol, and thyrotropin. J Clin Endocrinol Metab [Internet]. 2004 [acesso em 08 mar 2017];89(11):5762-71. Disponível em: http://dx.doi. org/10.1210/jc.2004-1003

27. Buxton OM, Quintiliani LM, Yang MH, Ebbeling CB, Stoddard $A M$, et al. Association of sleep adequacy with more healthful food choices and positive workplace experiences among motor freight workers. Am J Public Health. [Internet]. 2009 [acesso em 08 mar 2017];99(Suppl. 3):s636-43. Disponível em: http://dx.doi.org/10.2105/AJPH.2008.158501

28. Sahu S, Dey M.Changes in food intake pattern of nurses working in rapidly rotating shift. Al Ameen J Med Sci [Internet]. 2011 [acesso em 08 mar 2017];4(1):14-22. Disponível em: http:// connection.ebscohost.com/c/articles/57210267/changesfood-intake-pattern-nurses-working-rapidly-rotating-shift

29. Barboza JIRA, Moraes EL, Pereira EA, Reimão RNAA. Avaliação do padrão de sono dos profissionais de enfermagem dos plantões noturnos em Unidades de Terapia Intensiva. Einstein (São Paulo) [Internet]. 2008 [acesso em 08 mar 2017];6(3):298330. Disponível em: http://apps.einstein.br/revista/arquivos/ PDF/927-v6n3aA0927portp296-301.pdf

30. Maynardes DCD, Sarquis LMM, Kirchhof ALC. Trabalho noturno e morbidades de trabalhadores de Enfermagem. Cogitare Enferm. [Internet]. 2009 [acesso em 08 mar 2017];14(4):703-8. Disponível em: http://www.revenf. bvs.br/scielo.php?script=sci_arttext \& pid=S141485362009000400014\&lng=es.

31. Crispim CA, Zimberg IZ, Datillo M, Padilha HG, Tufik S, Mello MT. Trabalho em turnos e aspectos nutricionais: uma revisão. Nutrire Rev Soc Bras Aliment Nutr [Internet]. 2009 [acesso em 08 mar 2017];34(2)213-27. Disponível em: http://files.bvs.br/ upload/S/1519-8928/2009/v34n2/a213-227.pdf 